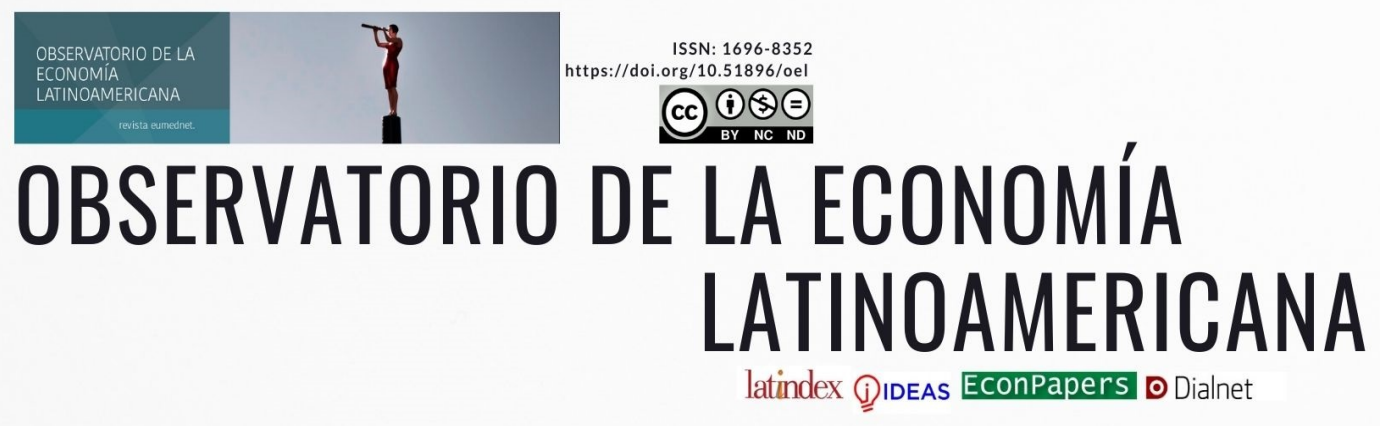

\title{
EL IMPACTO DE COVID-19 EN LAS EMPRESAS DE MÉXICO
}

Héctor Ruiz Ramírez ${ }^{1}$

Para citar este artículo puede utilizar el siguiente formato:

Héctor Ruiz Ramírez: "El impacto de Covid-19 en las empresas de Mexico.", Revista Observatorio de la Economía Latinomericana (Vol 19, № 4, pp. 79-95, abril-junio 2021). En línea:

https://doi.org/10.51896/oel/MTTA8939

\section{RESUMEN}

La pandemia derivada del COVID-19 ha marcado un fuerte impacto en prácticamente todas las naciones. La empresa es el lugar de trabajo y de sustento de miles de personas. Por ello resulta importante el conocer los efectos del virus en su fuente de trabajo. Este artículo pretende analizar el efecto del COVID-19 en la empresa mexicana, para lo cual se utilizan estadísticas de los momentos anteriores y posteriores a esta crisis, que en su origen fue de salud. Se aplica el método deductivo que contiene un enfoque cuantitativo descriptivo. Con tal fin, se utiliza la información que sobre el tema ha elaborado el Instituto Nacional de Geografía y Estadística (INEGI). Se concluye que la afectación ha llevado a la desaparición de miles de empresas y puestos de trabajo en prácticamente todos los sectores y en todo tipo de empresa.

Palabras clave: COVID-19, efectos de la pandemia, empresa mexicana, empleo, censos económicos.

\section{THE IMPACT OF COVID-19 ON COMPANIES IN MEXICO}

\section{ABSTRACT}

The pandemic derived from COVID-19 has had a strong impact on practically all nations. The company is the place of work and livelihood for thousands of people. For this reason, it is important to know the effects of the virus in your workplace. This article aims to analyze the effect of COVID-19 on Mexican companies, using statistics from the moments before and after this crisis, which was originally health. The deductive method that contains a descriptive quantitative approach is applied. To this end, the information prepared by the National Institute of Geography and Statistics (INEGI) is

\footnotetext{
${ }^{1}$ Profesor de la Facultad de Economía de la UAEMex. uaemherura@yahoo.com.mx
} 
used. It is concluded that the affectation has led to the disappearance of thousands of companies and jobs in practically all sectors and in all types of companies

Key Words: COVID-19, effects of the pandemic, Mexican company, employment, economic censuses.

Clasificación JEL: L25; M21

UNESCO: $530407 ; 531109 ; 531211$

\section{INTRODUCCIÓN}

La pandemia que se ha presentado debido a la aparición del COVID-19 ha provocado fuertes impactos no solamente en el ámbito de la salud, sino en gran parte de los entornos del ser humano. Su propagación mundial ha abarcado de igual manera a prácticamente todos los países del orbe, provocando enfermedad, mayor pobreza y desigualdad y en general menor bienestar en las familias, entre otros muchos efectos.

En el renglón económico ha llevado a fuertes caídas en la producción mundial, elevando considerablemente las tasas de desempleo al verse interrumpida la actividad productiva, lo que ha llevado a la empresa a no resistir en muchos casos esta situación, provocando su desaparición.

En la última semana de marzo de 2020, México emite una alerta debido a la aparición y propagación del Covid-19, por lo que se inicia una etapa de confinamiento por motivos sanitarios, que inmediatamente afecta al mundo de la producción, el trabajo y el bienestar de las personas.

Con relación a las condiciones de salud de la población mexicana para enfrentar al COVID-19, cabe señalar que en 2020 existían en México 20.2 millones de personas sin acceso a los servicios de salud; 71.7 millones de personas sin acceso a la seguridad social, donde el $75.2 \%$ padecía sobrepeso u obesidad y una décima parte diabetes. (CONEVAL, 2020).

En el presente artículo se pretende analizar la afectación e impacto que ha tenido la empresa mexicana ante la aparición y diseminación del COVID-19, con la información estadística nacional disponible, más reciente.

\section{METODOLOGÍA}

La investigación tiene un enfoque cuantitativo y es de tipo no experimental. Al tener un enfoque cuantitativo, necesariamente el método que se utiliza es el Método Deductivo, que va de lo general a lo particular. Se aplica el análisis descriptivo con el apoyo de gráficas elaboradas para tal fin.

Básicamente se utiliza información generada por el Instituto Nacional de Estadística y Geografía (INEGI) que es la institución encargada de normar y coordinar el Sistema Nacional de Información Estadística y Geográfica en México.

El INEGI es el organismo encargado del levantamiento de los Censos Económicos en México, los cuales se llevan a cabo cada cinco años. Recientemente el Instituto ha dado a conocer los resultados de los XIX Censos Económicos de 2019, con información referente al año anterior. Esta 
información es de mucha utilidad para el objetivo del presente artículo ya que muestra información inmediatamente anterior al surgimiento de la pandemia, lo que permite tener un panorama de la empresa mexicana antes del COVID-19.

Se utiliza la información de "La Encuesta sobre el Impacto Económico Generado por COVID-19 en las Empresas (ECOVID-IE) 2020" elaborada por el INEGI, cuyo fin es la medición del impacto del COVID-19 en la empresa mexicana (INEGI, 2021a).

Así mismo, se acude al "Estudio sobre la Demografía de los Negocios (EDN)", encuesta elaborada también por el INEGI, que proporciona información de las principales características de los establecimientos económicos manufactureros, comerciales y de servicios privados no financieros, que nacen y mueren (INEGI, 2021b).

\section{MARCO TEÓRICO}

Ante lo nuevo de este fenómeno ha iniciado la aparición de estudios e investigaciones que tratan de observar y medir las consecuencias de la pandemia. El Banco Mundial (2020) señala que a pesar de la literatura del COVID-19 en rápida expansión, no hay evidencia sistemática sobre el impacto de la pandemia en las empresas, ya que solamente se focaliza en pequeñas muestras y en empresas seleccionadas. En su encuesta encontró que la probabilidad de que una empresa continúe abierta en el pico de la crisis de COVID-19 y hasta 4 semanas después del pico, está por debajo del 30\%, pero aumenta a casi el $75 \%$ o más, 6 semanas después del pico.

En la "Encuesta sobre la Actividad Empresarial" del Banco de España se registra que la crisis sanitaria del COVID-19 es de tipo asimétrica, tanto a nivel sectorial, como regional, ya que el impacto ha tenido efectos más nocivos en las empresas más jóvenes, las de menor nivel de productividad y las ubicadas en áreas urbanas (BE, 2021).

El COVID-19 ha impactado negativamente en la empresa fundamentalmente en el área de ventas, empleo, restricción del crédito, teniéndose una fuerte incertidumbre sobre el futuro (BM, 2020).

El Banco Mundial afirma que en $25 \%$ de las empresas las ventas han caído a la mitad y que en aproximadamente el $65 \%$ se redujeron salarios, ya sea mediante su disminución o a través de licencias o menos horas de trabajo, lo que llevó a que aproximadamente solo una décima parte de las empresas despidiera personal. En cuanto al mayor uso de infraestructura digital solamente una tercera parte ha usado más el internet, redes sociales y plataformas digitales (BM, 2021a).

Di Mauro y Syverson (2020) señalan que las ventas han disminuido en alrededor del $84 \%$ de las empresas en todos los países. El promedio entre países alcanza una baja de aproximadamente la mitad. También la mitad de las empresas hicieron un mayor uso de la tecnología y de plataformas digitales. Alrededor de una vigésima parte buscaron diversificarse hacia productos o servicios relacionados con la salud (Di Mauro y Syverson, 2020; citados en BM, 2020).

Se ha demostrado que la comercialización digital permite lograr resultados muy favorables, garantizando la atracción de nuevos clientes y aumentos sustanciales en las ventas de cualquier 
empresa. Los negocios que comenzaron a actualizar al nuevo modelo de comercio digital han tenido un fuerte incremento en ventas y nuevos clientes, obligados por el impacto de COVID-19 (Verastegui \& Vargas, 2021).

En una investigación donde se analiza el volumen y los patrones de gasto de los consumidores, los volúmenes destinados a la obtención de bienes de consumo específicos muy demandados y en bienes de lujo y no duraderos, se concluye que el comportamiento del consumidor durante la crisis del COVID-19 parece alinearse con los comportamientos exhibidos durante eventos históricos de choque (Loxton:et.al, 2020).

En un estudio cuyo objetivo fue analizar el enfoque de marketing estratégico de Starbucks Coffee, se concluye que esta empresa debería aumentar la distribución de productos que tienen más probabilidades de tener éxito durante la pandemia de COVID-19, y expandir los servicios de recogida y entrega (Lombardi, Chidiac, \& Record, 2021).

El Centro del Comercio Internacional $(\mathrm{CCl})$ resultado de su "Encuesta sobre el Impacto Empresarial de la COVID-19 del ITC" afirma que una quinta parte de la pequeña empresa podría entrar en quiebra en tres meses. El COVID-19 ha afectado a aproximadamente el 66\% de las micro y pequeñas empresas y a dos quintas parte de la gran empresa. También consideran que el sector de servicios ha sido el más afectado $(\mathrm{CCl}, 2020)$.

En un estudio sobre Pymes se hace énfasis en que en una pandemia como la actual se requiere una gran movilización de recursos, en donde ni los sistemas financieros, ni los empresarios estaban preparados (Kahveci, 2021).

Se ha medido el impacto de la pandemia de COVID-19 en las Pymes de Armenia, encontrándose que, en comparación con los empleados de las grandes empresas, las más pequeñas se ven mayormente afectadas por la crisis, ya que ha habido despidos o han reducido su jornada laboral y su salario. Ser trabajador de una PYME duplica la probabilidad de despido y reducción salarial. Los empleados en empresas donde se permite el trabajo a distancia, como el caso de la educación y los servicios de información y comunicación, se han visto menos vulnerables por la crisis (Beglaryan \& Shakhmuradyan, 2020).

Demuner Flores en una encuesta a empresarios de Córdoba Veracruz, en México, cuyo objetivo fue analizar el uso de redes sociales ante los efectos del COVID-19, encontró que aproximadamente la mitad de las microempresas fueron afectadas por el cierre temporal obligatorio, provocando la caída en sus ingresos (31\%) y despidiendo trabajadores (16\%), por lo que el 30\% decidió aumentar el uso de redes sociales (Demuner, 2021).

Se ha investigado si las empresas que cotizan en la Bolsa de Valores de Bucarest se han preparado para el riesgo del Covid-19 y cuáles son las medidas e iniciativas tomadas por ellas en términos de sostenibilidad. Se descubrió que la mayoría de las empresas no han estado preparadas para tal riesgo, a pesar de lo cual respondieron de inmediato y han tomado medidas para proteger a sus empleados (Stoica, 2020). 
Se evaluó la reacción de los principales mercados bursátiles asiáticos ante el brote temprano de la pandemia de COVID-19 y sus efectos secundarios entre estos mercados. El hallazgo indica evidencia de que cuando no se comprendió plenamente la importancia de la pandemia, existía un fuerte vínculo e influencia entre estos mercados, pero cuando la escala y el tamaño de la pandemia fue mayor, se dieron cuenta de que estos mercados mostraban un comportamiento de disociación (Onour, 2021).

Un estudio realizado en Rumania en empresas grandes y pequeñas que cotizan en Bolsa y que pertenecen a diferentes sectores comerciales tuvo como objetivo analizar los cambios clave en las actividades para evaluar el nivel de desempeño empresarial en respuesta a la pandemia COVID-19, encontrándose que los beneficios netos del mercado disminuyeron $37.43 \%$. Las pequeñas empresas dedicadas a la agricultura, el comercio, la construcción, la I + D de Tl y el transporte y almacenamiento, obtuvieron mejores resultados financieros (Achim \& et.al, 2021).

En un estudio de empresas bancarias se encontró que la banca de desempeño financiero no experimentó ninguna diferencia antes y durante Covid-19 por lo que se concluyó que los bancos aún pueden mantener la estabilidad financiera bancaria durante una pandemia (Wardhani, Elvany, \& Awaluddin, 2021).

Una encuesta realizada a personas en Jabodetabek, que es el área metropolitana de Yakarta en Indonesia, para analizar si la autoeficacia, la motivación intrínseca y la motivación extrínseca pueden influir en el desempeño de los empleados, encontró que, a mayor autoeficacia en el trabajo, aumenta el rendimiento del empleado. La motivación que afecta el desempeño de los empleados es extrínseca y no intrínseca. En una pandemia como la actual, la motivación extrínseca debe centrarse más en crear programas atractivos de incentivos, bonificaciones, comisiones, estimular a los empleados que han completado las tareas y reconocer su buen trabajo (Nilasari, Nisfiannoor, \& Devinta, 2021).

En un estudio realizado en un banco gubernamental en Kediri, Java Oriental, Indonesia cuyo objetivo fue determinar el efecto de la colocación y motivación laboral en el desempeño de los empleados, se detectó que la colocación de los empleados y la motivación laboral influyen en su desempeño, eficacia y productividad (Suswati, 2021).

En una investigación cuyo objetivo fue el de identificar si la crisis de COVID-19 ha tenido un impacto en la comunicación interna de la empresa, tanto desde la perspectiva del líder como de los miembros de la organización. Se concluye que debido a la pandemia el modo presencial de comunicación se reemplazó por el modo virtual (Bojadjiev \& Marjana, 2021).

Ante la aparición del COVID-19 en empresas de Indonesia se ha analizado cómo la implementación del tipo de innovación frugal está influenciada por el estilo de liderazgo transformacional. Los hallazgos muestran que el líder transformacional, el equipo de gestión de crisis, la calidad de la comunicación y el tipo de innovación frugal, influyeron positivamente en la eficacia de la gestión de crisis (Dwiedienawati \& et.al, 2021). 
En un análisis estadístico de los aspectos de seguridad de las TIC se mostró la importancia de invertir recursos en ciberseguridad en la actual situación pandémica generada por COVID-19 (Meghisan-Toma \& Cosmin, 2020).

\section{LA EMPRESA MEXICANA ANTES DEL COVID-19}

Para conocer la situación de la empresa mexicana antes del Covid-19 se utiliza la información del último Censo Económico de México, el cual se levantó en el periodo de febrero a julio de 2019, captando información de la actividad realizada durante todo el año de 2018 y de los establecimientos económicos que iniciaron actividades en el periodo del levantamiento censal.

Este apartado se apoya básicamente en la estadística obtenida en los XIX Censos Económicos de 2019 levantados por el INEGI, donde se incluyen a los sectores Manufacturero, Comercio, Servicios; Construcción; y Transportes, correos y almacenamiento y Otras actividades económicas, tales como Pesca y acuicultura; Minería; Electricidad, agua y gas (INEGI, 2020c). No se incluyen las actividades agropecuarias y forestales, ya que son objeto de un censo aparte.

La unidad de observación que se utiliza en los censos económicos mexicanos es el "establecimiento", por lo que hay que considerar que una empresa puede estar conformada por varios de ellos, por lo que teóricamente el número de empresas será menor al de establecimientos.

De esta forma, de los 4,800,157 establecimientos captados en 2018 pertenecientes al Sector privado y paraestatal, se conformaron 4,616,864 empresas (INEGI, 2020c).

Del total de establecimientos, 6,044,821 iniciaron operaciones antes del año de 2019 y 328,348 lo hicieron durante 2019 (INEGI, 2020c).

\section{Gráfica 1.}

Número de establecimientos en México 2004-2019

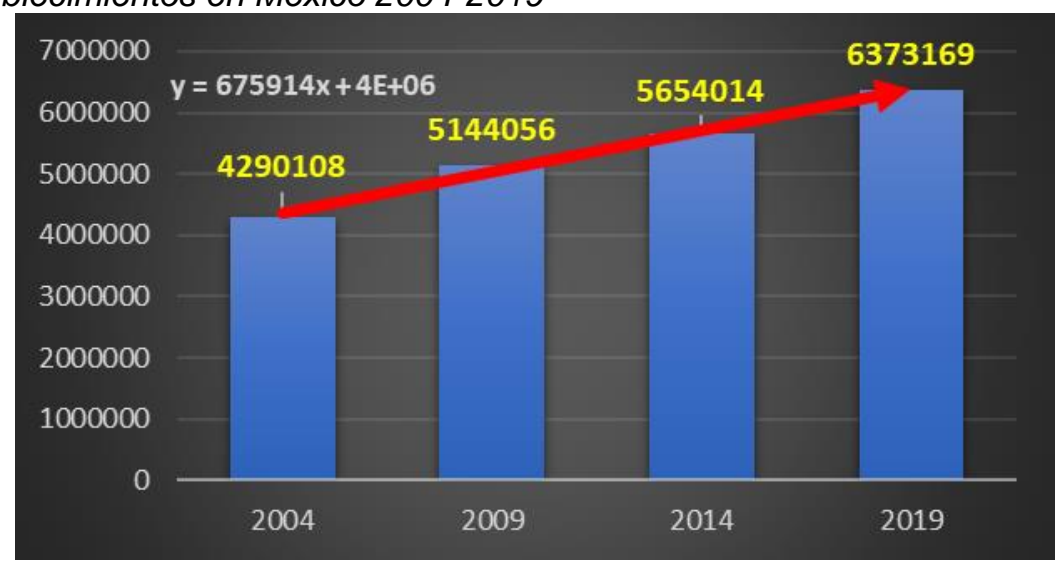

Fuente: elaboración propia con base en (INEGI, 2020a)

En 2019 el número de establecimientos censados en México fue de 6,373,169. Al compararse con el dato de 2014 esta cifra se incrementó en 719,155 establecimientos, lo que significa un crecimiento medio anual de $2.4 \%$. Y se incrementa en 1,229,113 con relación a 2009. En los últimos tres lustros el crecimiento en el número de establecimientos fue de 2,083,061. 
El crecimiento más alto en el número de establecimientos en los últimos 15 años corresponde al periodo que va de 2004 a 2009 con una tasa promedio anual de $3.7 \%$ y el más bajo de 2009 a 2014, con un $1.9 \%$ (gráficas 1 y 2 ).

\section{Gráfica 2.}

Tasa de crecimiento del número de establecimientos en México para los periodos que se indican

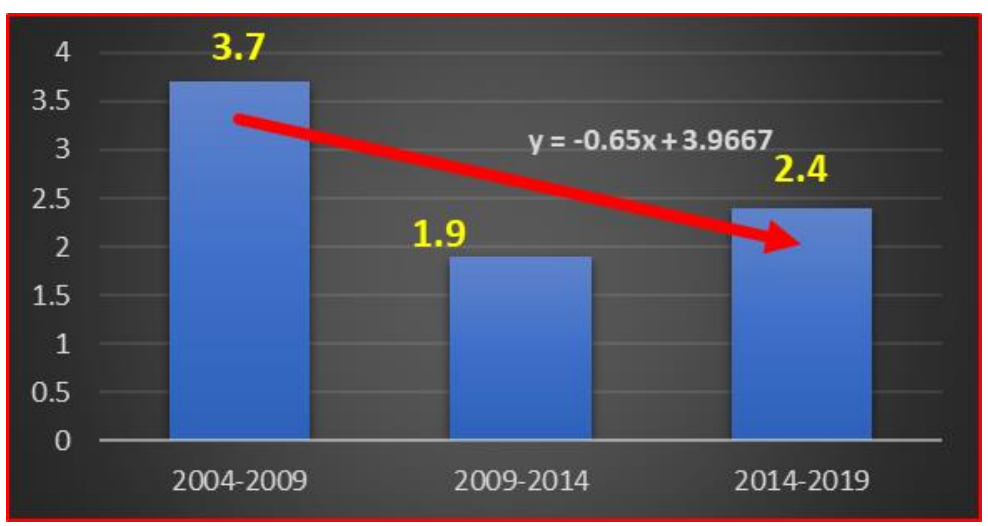

Fuente: elaboración propia con base en (INEGI, 2020a)

En los 6,373 millones de establecimientos que registró el censo, laboraban 36,038.272 trabajadores. Esta cifra de empleo es 6,395,851 superior a la registrada cinco años atrás, en 2014, lo que significa un crecimiento promedio anual de 1,279,170 personas en el periodo de referencia.

\section{Gráfica 3.}

Personal ocupado en los establecimientos en México 2004-2019

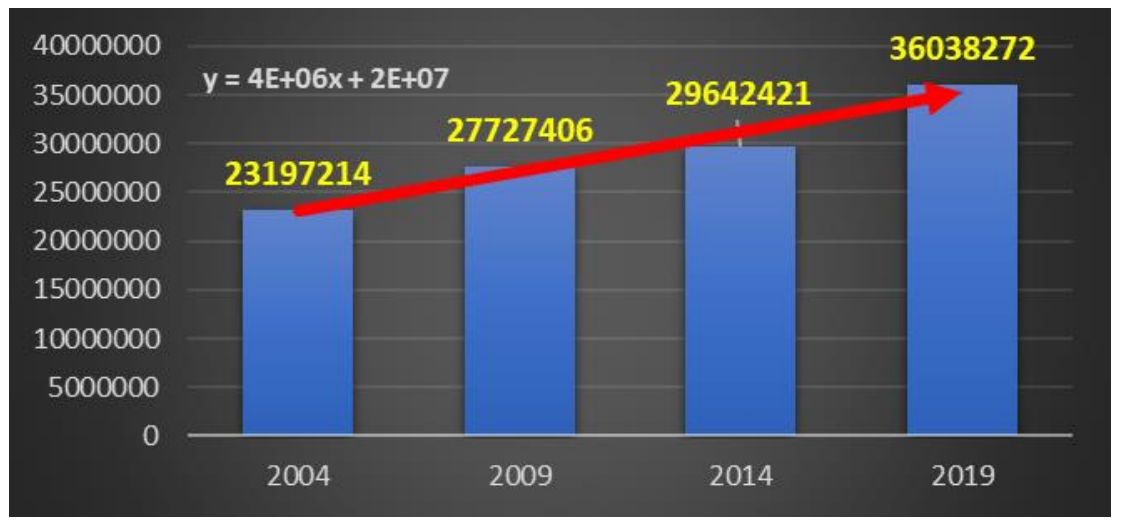

Fuente: elaboración propia con base en (INEGI, 2020a)

De acuerdo con la gráfica 4, la tasa media anual de crecimiento del empleo en los establecimientos censados fue de $4.0 \%$, en el periodo de 2014 a 2019. La tasa más baja se observa de 2009 a 2014 , con $1.3 \%$ y la correspondiente al periodo de 2004 a 2009 , fue de $3.6 \%$.

Resalta el hecho de que durante el periodo de 2004 a 2009 con una tasa de crecimiento medio anual de 3.7\% el empleo se incrementó en 3.6\%, mientras que el periodo de 2014 a 2019 con una 
tasa de crecimiento medio anual en establecimientos de $2.4 \%$, el personal reflejó un aumente de $4.0 \%$, lo que significa probablemente un mayor uso intensivo de mano de obra.

\section{Gráfica 4.}

Tasa de crecimiento del Personal Ocupado en los establecimientos en México para los periodos que se indican

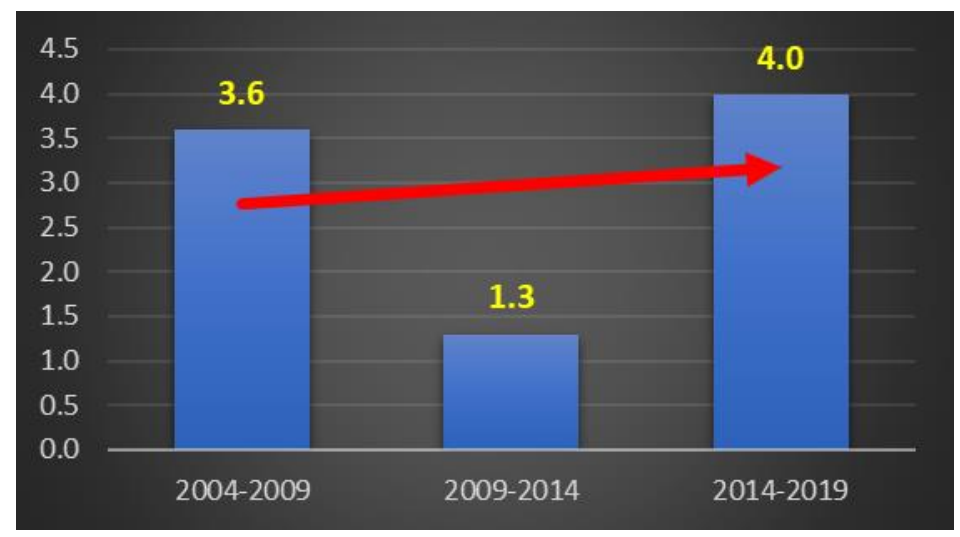

Fuente: elaboración propia con base en (INEGI, 2020a)

Otros datos que arroja el XIX Censo Económico y que permiten tener una mejor visión de la empresa mexicana antes del Covid-19, son los siguientes (INEGI, 2020a):

$\checkmark$ Del total de establecimientos censados, el $62.6 \%$ pertenecen al sector informal, trabajando en este tipo de establecimientos el $18.9 \%$. El sector formal lo integran el $37.4 \%$ de unidades económicas con el $81.1 \%$ del total de personas ocupadas. En cuanto al valor agregado los formales contribuyeron con el $97 \%$ y los informales con el restante $3 \%$.

$\checkmark$ Los grandes establecimientos solamente representaron el $0.2 \%$ del total, mientras que los medianos y pequeños (Pymes) el $4.9 \%$ y los denominados micro, el $94.9 \%$. Una estructura muy similar que se encuentra en la mayoría de los países.

$\checkmark$ Resalta el hecho de que las Pymes contribuyen con el $30.7 \%$, tanto del valor agregado como del personal que se ocupa. En establecimientos grandes con el $32.1 \%$ y micronegocios con $37.2 \%$.

$\checkmark$ Del total de Pymes, el $86.3 \%$ cuenta con equipo de cómputo, pero a pesar de este alto porcentaje, solamente el $18.7 \%$ realizó ventas vía internet. En los establecimientos grandes no cambia mucho esta participación ya que el $24 \%$ utilizó el internet con este fin.

$\checkmark$ A nivel sectorial los establecimientos dedicados a la manufactura participan con el $32 \%$ del valor agregado total, la minería con el $9.5 \%$, electricidad con $2.2 \%$, el comercio con $21.4 \%$ y las actividades restantes con $14.3 \%$.

$\checkmark$ Del universo de empresas, las denominadas Micro representaron el 96\%, proporcionando empleo al $35 \%$ del total ocupado y generando el $8.3 \%$ del producto bruto total. Del lado opuesto la Gran Empresa representó el 0,2\% del total de empresas, empleando al $42.7 \%$ del total, con una producción del $73,4 \%$. Mientras que la micro generan menos de una 
décima parte de la producción, las grandes empresas aportan casi tres cuartas partes. (INEGI, 2020c).

\section{LOS EFECTOS DEL COVID-19 EN LA EMPRESA MEXICANA}

\subsection{Afectación por tipo de empresa}

El Estudio sobre la Demografía de los Negocios (EDN), del INEGI, brinda información de las principales características de los establecimientos económicos manufactureros, comerciales y de servicios privados no financieros, que nacen y mueren.

Los establecimientos censados mencionados en el párrafo anterior arrojaron un total de 4,857,007. De mayo de 2019 a septiembre de 2020, que incluye parte del periodo de pandemia, se registran 619,443 nacimientos de empresas y 1,010857 de muertes, por lo que al final del se tiene un nuevo total de 4,465,593 (gráfica 5). Ello significa un decremento de $8.06 \%$.

Dado que la tendencia en el crecimiento del número de empresas antes de la pandemia era ascendente (gráfica 1) es posible deducir que buena parte de la muerte de estas empresas se debió a los efectos del confinamiento y que por cuestiones de mercado y financieras no les fue posible superar esta etapa.

Se estima que los establecimientos de tipo micro tuvieron mayores muertes de empresas con el $20.8 \%$ y las Pymes (pequeñas y medianas) de 21.2\% (INEGI, 2020b).

\section{Gráfica 5.}

Número de establecimientos en México. Mayo de 2019 y septiembre de 2020

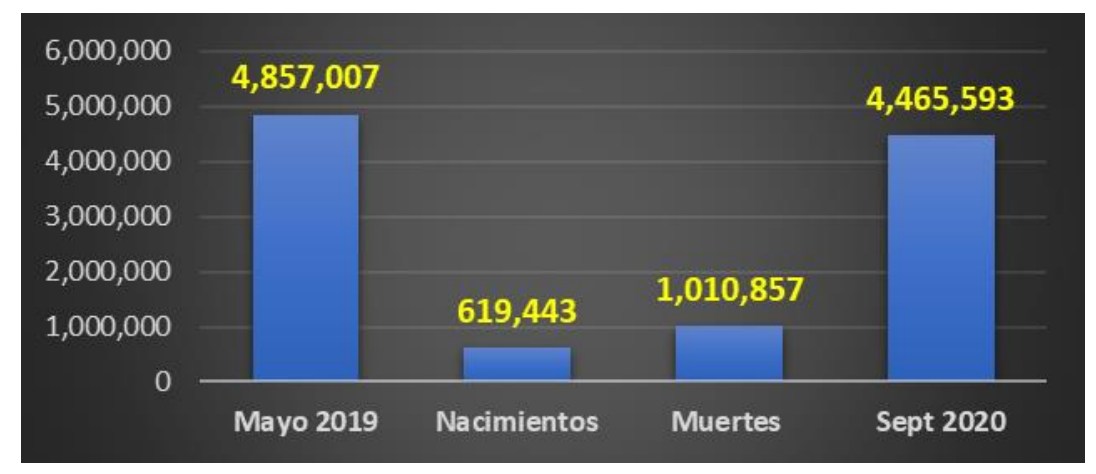

Fuente: elaboración propia con base en (INEGI, 2021b)

Por tipo de actividad económica, en el periodo de mayo de 2019 a septiembre de 2020 , donde se presentó el mayor porcentaje de establecimientos muertos fue en el de Servicios Privados no Financieros con el 25\%, siguiéndole el de Comercio con 19\% y Manufacturas con 15\%.

El Estudio encontró que los establecimientos nacidos durante 2020, año de inicio de la pandemia, registraron en promedio dos personas ocupadas y los que cerraron, tenían en 2018 un promedio de tres personas, cifra semejante a la que mantuvieron los sobrevivientes (INEGI, 2021b). 
La "Encuesta sobre el Impacto Económico Generado por COVID-19 en las Empresas (ECOVID-IE)" realizada por el INEGI en tres momentos diferentes, tiene el objetivo de "Conocer la situación actual y afectaciones en las empresas por la contingencia originada por COVID-19)" (INEGI, 2021c).

\section{Gráfica 6.}

Porcentaje de afectación del Covid-19 en la empresa mexicana, según evento*

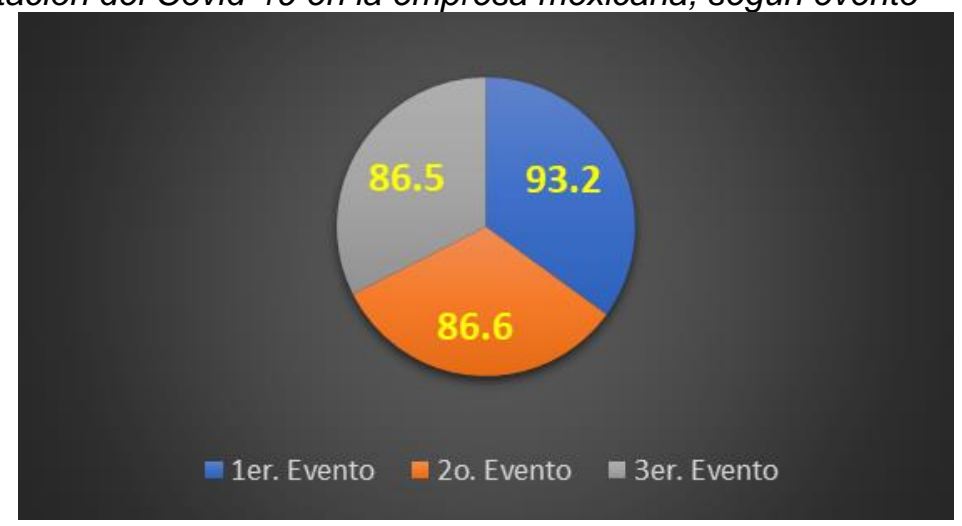

*Primer evento: abril 2020; segundo evento: agosto 2020; tercer evento: febrero 2021

Fuente: elaboración propia con base en (INEGI, 2021c).

Se observa en la gráfica 6 que de acuerdo con los tres momentos en que el INEGI (2021c) capta información sobre la afectación del COVID-19 en la empresa mexicana, fue en el momento inicial, en abril del año 2020, cuando la gran mayoría de empresas tuvo cierres temporales o disminución en su actividad productiva, con lo que se consideró afectada el $93.2 \%$ del total de las empresas. En los siguientes eventos de agosto de 2020 y de febrero de 2021 se tuvo una leve disminución del nivel de afectación, al registrarse casi idéntico porcentaje de $86.6 \%$ y $85.5 \%$ respectivamente.

\section{Gráfica 7.}

Porcentaje de empresas mexicanas afectadas por baja demanda

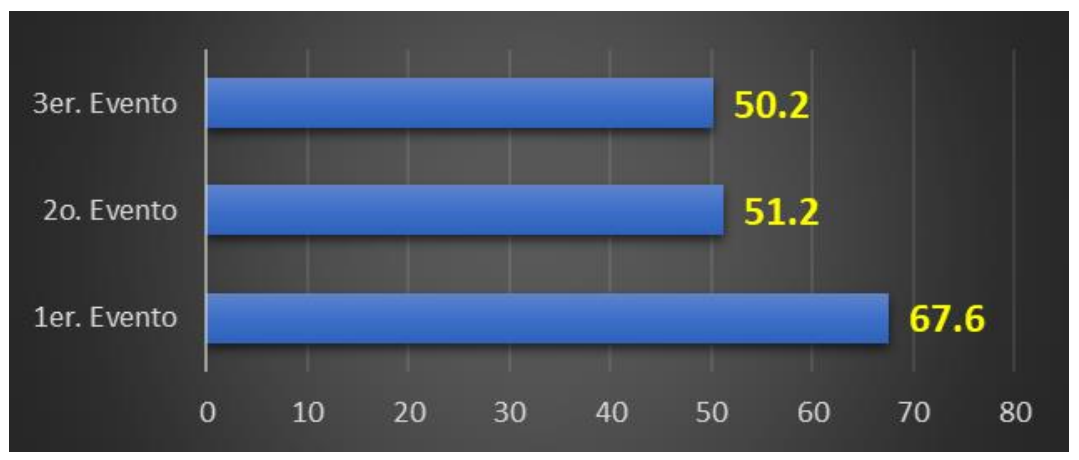

*Primer evento: abril 2020; segundo evento: agosto 2020; tercer evento: febrero 2021

Fuente: elaboración propia con base en (INEGI, 2021c).

En la gráfica 7 se registra que fue en el primer evento donde la empresa mexicana resintió con mayor fuerza una disminución en la demanda con el $67.6 \%$ del total. En los siguientes dos eventos hay una sensible disminución a $51.2 \%$ en el segundo evento y a $50.2 \%$ en el primer evento, lo que significó una mejoría en la demanda. 


\section{Gráfica 8.}

Porcentaje de afectaciones en las Grandes Empresas Mexicanas, por evento

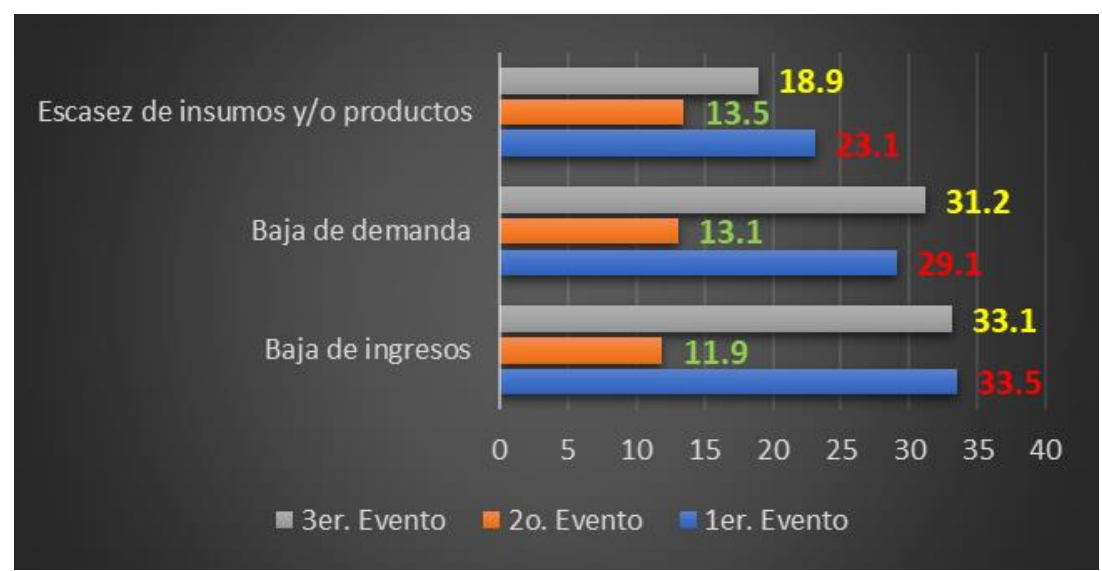

*Primer evento: abril 2020; segundo evento: agosto 2020; tercer evento: febrero 2021

Fuente: elaboración propia con base en (INEGI, 2021c).

La gráfica 8 muestra la medición de los principales efectos del COVID-19 en las Grandes Empresa de México. La disminución en los ingresos se observa preponderantemente en el primer (33.5\%) y tercer evento (33.1\%). En cuanto a la baja en la demanda, nuevamente es en el primer evento (29.1\% y el tercero $(31.2 \%)$ donde se registra la mayor afectación. Con relación a la escasez de insumos y/o productos, la mayor afectación se encuentra en el primer evento (23.1\%) y en el tercero (18.9\%). En los tres indicadores que se miden, es en el segundo, donde la empresa grande percibió una menor afectación.

\section{Gráfica 9.}

Adopción permanente de medidas operativas de la empresa mexicana ante el COVID-19

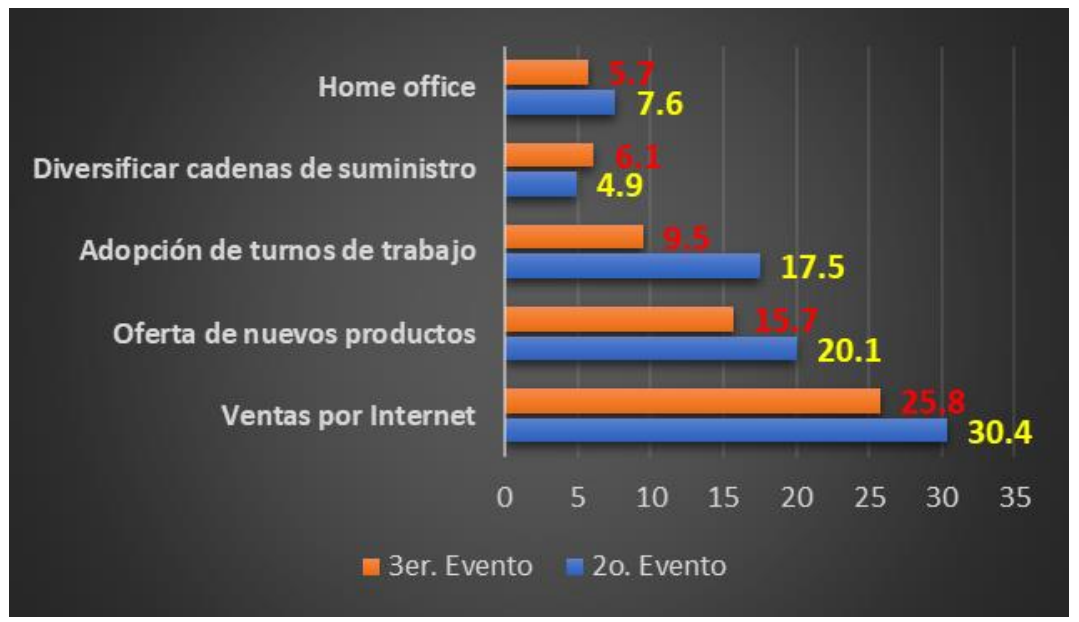

*Segundo evento: agosto 2020; tercer evento: febrero 2021

Fuente: elaboración propia con base en (INEGI, 2021c).

En la gráfica 9 se observan las medidas que las empresas mexicanas pretenden implantar de manera permanente ante los efectos del COVID-19. Excepto la diversificación de las cadenas de suministro, en los restantes indicadores siempre el porcentaje es mayor en el segundo evento que 
en el tercero. Esto pudiera ser efecto de que la empresa mexicana ha empezado a asimilar los efectos negativos del COVID-19 y que conforme pasa el tiempo sus expectativas de cambio debido a ello, son menores, aunado a que hay mejores condiciones para la reapertura.

Tal vez se hubiese esperado un mayor deseo por realizar ventas por internet, el ofrecer nuevos productos o el trabajo en casa.

\section{Gráfica 10.}

Porcentaje de Nacimientos y Muertes de Establecimientos en México. Mayo 2019-Septiembre 2020

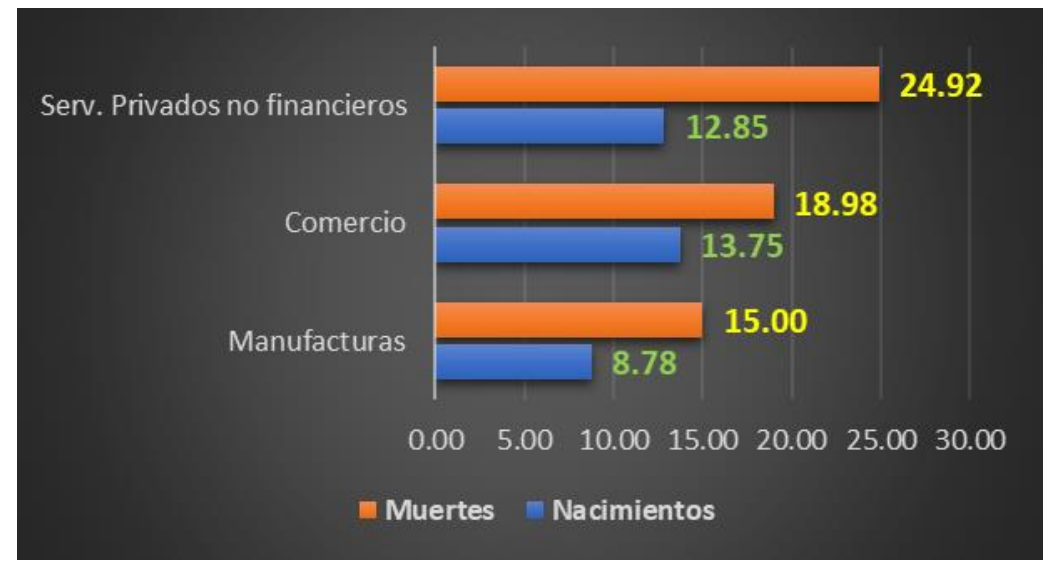

Fuente: elaboración propia con base en (INEGI, 2020b)

Del total de establecimientos que murieron (20.8\%) el sector informal registró el $12.8 \%$ y el $5.6 \%$ del sector formal. Mientras que del total de establecimientos que sobrevivieron (79.2\%) el $46.6 \%$ pertenece al sector informal y el $25.9 \%$ al formal (gráfica 11 ).

\section{Gráfica 11.}

Porcentaje de establecimientos muertos y sobrevivientes del Sector Formal e Informal en México. Mayo 2019 - Septiembre 2020*

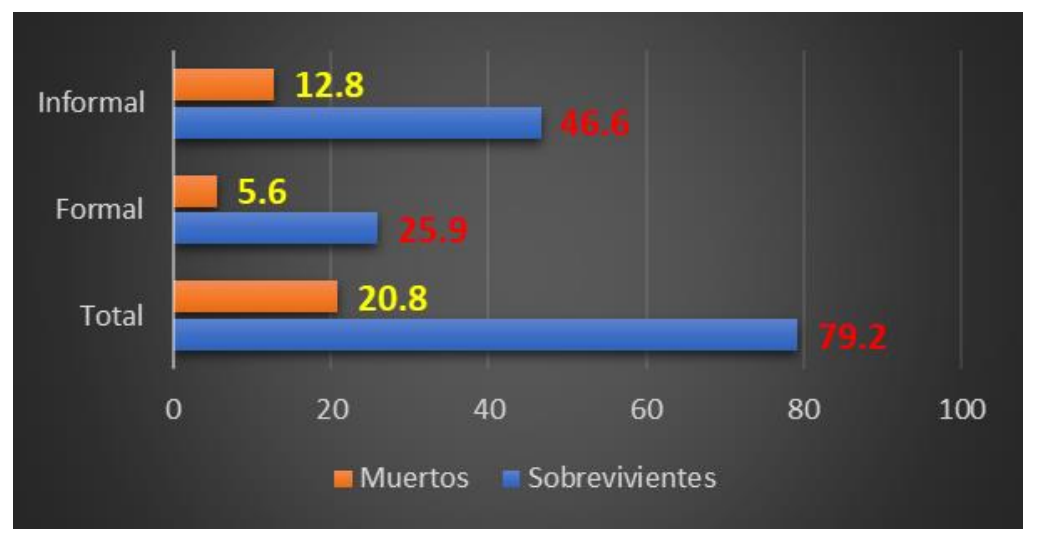

*Excluye $2.4 \%$ de establecimientos muertos y $7.3 \%$ de sobrevivientes en los que no fue posible identificar su condición de informalidad o formalidad.

Fuente: elaboración propia con base en (INEGI, 2021b) 


\subsection{Efectos en el empleo}

En cuanto al efecto de la pandemia en la ocupación, en mayo de 2019 laboraban, de acuerdo con el Censo Económico 14,660,209 personas y para septiembre de 2020, este total se redujo a $11,775,047$. En los establecimientos que desaparecieron laboraban 2,966,965 trabajadores; pero aún en los que lograron sobrevivir se registró una pérdida de 1,149,494, quedando para el mes de mayo de 2020 un total de 11,775,047 trabajadores, por lo que se dio una disminución de 2,885,162 en el periodo de referencia.

Otro hallazgo del estudio del INEGI (2021b) mostró que la mayor cantidad de empleo perdido se dio en el bimestre de abril y mayo de 2020, tanto en el sector informal como en el formal, con una disminución de $27 \%$ y $23 \%$ respectivamente (INEGI, 2021b).

\section{Gráfica 12.}

Personal Ocupado en los Establecimientos en México. Mayo 2019-Septiembre 2020

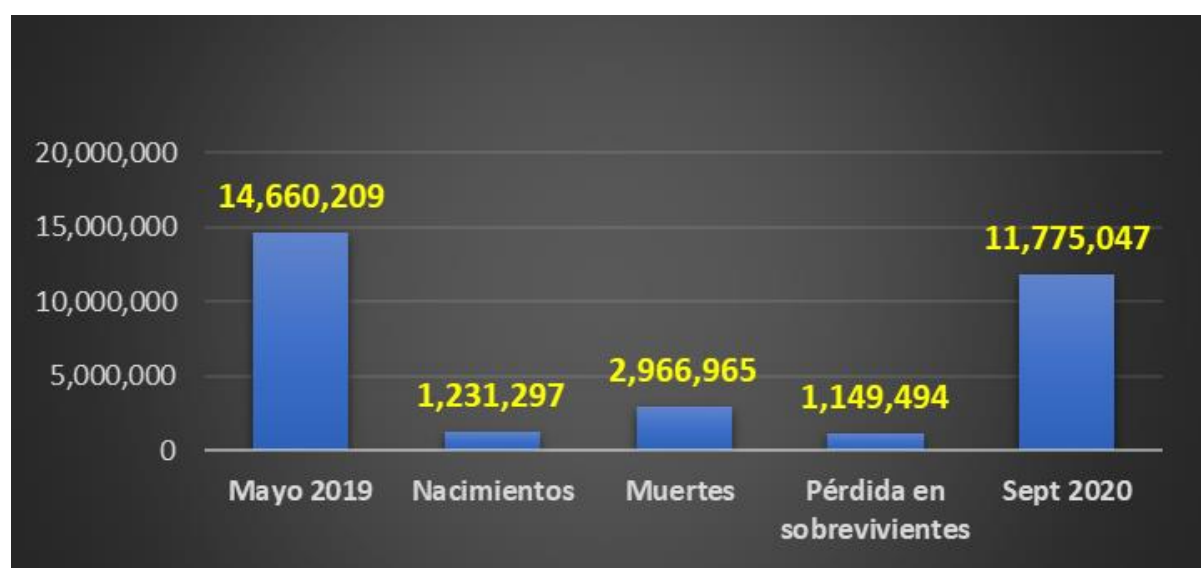

Fuente: elaboración propia con base en (INEGI, 2020b)

La estadística del Sector Formal del Empleo muestra que, en marzo de 2020, al inicio de la pandemia, se tenían registrados un total de 20,482,943 trabajadores. Para septiembre del mismo año ya se mostraba una disminución de 780,751 puestos de trabajo. Si se compara la cifra del inicio de la pandemia de marzo de 2020 con la de junio de 2021, a pesar de que ha existido una gran reapertura de negocios, aunque no en su totalidad, aún quedan por recuperarse 307,563 empleos, a los que habría que sumar los que se debieron de haber creado de no existir la pandemia (gráfica 13).

\section{Gráfica 13.}

Número de trabajadores en el Sector Formal en México, en los periodos que se indican 


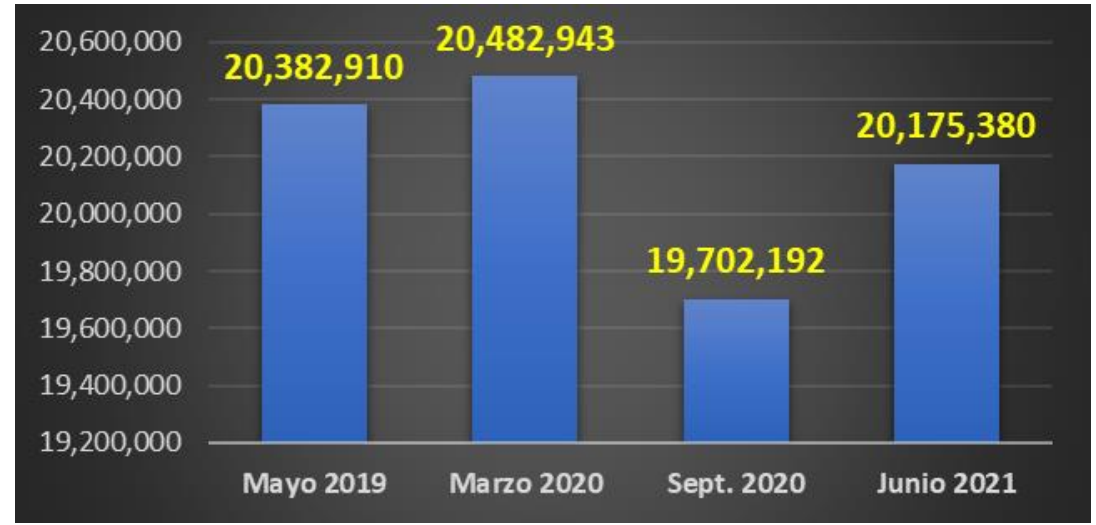

Fuente: elaboración propia con base en (STPS, 2021)

\section{CONCLUSIONES}

Ante un hecho sin precedentes recientes como la pandemia y el efecto que ha causado en la empresa mexicana y el empleo, resulta pertinente el iniciar el estudio del fenómeno, en principio con la información disponible y paralelamente con trabajo de campo, para poder diseñar mejores mecanismos para que a la mayor brevedad posible y una vez superada la crisis sanitaria, se pueda retomar el sendero del crecimiento productivo y la generación de empleo.

En México miles de empresas y empleos han desaparecido y el proceso de recuperación, seguramente será muy arduo. Sin embargo, como en toda crisis, también se pueden obtener aspectos positivos. Ha habido una reorientación en muchos casos hacia el teletrabajo, las ventas por internet, la educación a distancia, por mencionar algunos. Es muy probable que esquemas que se han ensayado quedarán de manera permanente y esto será una gran lección que permita aprender y adoptar mecanismos nuevos, en aras de una mejor productividad.

\section{REFERENCIAS}

Achim, M. V., Safta, I. L., Văidean, V. L., Mureșan, G. M., \& Borlea, N. S. (17 de mayo de 2021). The impact of covid-19 on financial management: evidence from Romania. Obtenido de Economic Research-Ekonomska Istraživanja:

https://www.tandfonline.com/doi/pdf/10.1080/1331677X.2021.1922090?needAccess=true

Banco de España (BE). (25 de febrero de 2021). El Impacto Económico del COVID-19 en las

Empresas Españolas según la EBAE. Obtenido de Banco de España:

https://repositorio.bde.es/bitstream/123456789/15199/1/be2101-art04.pdf

Beglaryan, M., \& Shakhmuradyan, G. (15 de septiembre de 2020). he impact of COVID-19 on small and medium-sized enterprises in Armenia: Evidence from a labor force survey. Obtenido de Small Business International Review. VOLUME: 4; ISSUE: 2; ELOCATION-ID: e298: https://doi.org/10.26784/sbir.v4i2.298

Banco Mundial (BM). (octubre de 2020). Unmasking the Impact of COVID-19 on Businesses. Firm Level Evidence from Across the World. Obtenido de Banco Mundial: 
https://openknowledge.worldbank.org/bitstream/handle/10986/34626/Unmasking-the-Impactof-COVID-19-on-Businesses-Firm-Level-Evidence-from-Across-the-World.pdf?sequence $=5$

Banco Mundial (BM). (17 de febrero de 2021a. Cómo la COVID-19 (coronavirus) afecta a las empresas en todo el mundo. Obtenido de Banco Mundial: https://www.bancomundial.org/es/news/infographic/2021/02/17/how-covid-19is-affectingcompanies-around-the-world

Bojadjiev, M. I., \& Marjana, V. (junio de 2021). The Impact of Covid-19 Crisis on a Company's Internal Communication. Obtenido de Journal Interdisciplinary Description of Complex Systems. Vol. 19, no. 2. pp. $239-256$ :

https://hrcak.srce.hr/index.php?show=clanak\&id_clanak_jezik=377602

Centro de Comercio Internacional (CCI). (2020). Perspectivas de competitividad de las PYMES. Covid-19 el gran confinamiento y su impacto en las pequeñas empresas. Obtenido de Centro de Comercio Internacional:

https://www.intracen.org/uploadedFiles/intracenorg/Content/Publications/SMECO2020/ITC_S MECO-2020ExSummary-Spanish.pdf

Consejo Nacional de Evaluación de la Política de Desarrollo Social (CONEVAL). (7 de mayo de 2020). La Política Social en el contexto de la Pandemia por el virus SAR-CoV-2 (COVID-19) en México. Obtenido de Consejo Nacional de Evaluación de la Política de Desarrollo Social: https://www.coneval.org.mx/Evaluacion/IEPSM/Documents/Politica_Social_COVID-19.pdf

Demuner, F. M. (2021). El uso de redes sociales en microempresas ante efectos COVID-19. Obtenido de Revista de Comunicación de la SEECI, 54, 97-118: https://doi.org/10.15198/seeci.2021.54.e660

Di Mauro, F., \& Syverson, C. (abril de 2020). The covid crisis and productivity growth. Technical report. VOXEU. Obtenido de Voxeu.

Dwiedienawati, D., Tjahjana, D., Faisal, M., Gandasari, D., \& Bramatoro, S. A. (29 de abril de 2021). Determinants of perceived effectiveness in crisis management and company reputation during the COVID-19 pandemic. Obtenido de Cogent Business \& Management, 8:1912523: https://www.tandfonline.com/doi/pdf/10.1080/23311975.2021.1912523?needAccess=true Instituto Nacional de Estadística y Geografía (INEGI). (16 de julio de 2020a). INEGI presenta los resultados definitivos de los Censos Económicos 2019. Obtenido de Instituto Nacional de Estadística y Geografía: https://www.inegi.org.mx/contenidos/saladeprensa/boletines/2020/OtrTemEcon/CenEconRes Def2019_Nal.pdf

Instituto Nacional de Estadística y Geografía (INEGI). (2 de diciembre de 2020b). EDN 2020 Estudio sobre la demografía de los negocios 2020. Primer conjunto de resultados. Obtenido de Instituto Nacional de Estadística y Geografía: https://www.inegi.org.mx/contenidos/programas/edn/2020/doc/EDN2020Pres.pdf Instituto Nacional de Estadística y Geografía (INEGI). (2020c). Las empresas en los Estados Unidos Mexicanos : Censos Eonómicos 2019. Obtenido de Instituto Nacional de Estadística y Geografía: 
https://www.inegi.org.mx/contenidos/productos/prod_serv/contenidos/espanol/bvinegi/product os/nueva_estruc/702825198817.pdf

Instituto Nacional de Estadística y Geografía (INEGI). (Abril de 2021a). Encuesta sobre el Impacto Económico Generado por COVID-19 en las Empresas (ECOVID-IE) 2020. Obtenido de Instituto Nacional de Estadística y Geografía: inegi.org.mx/programas/ecovidie/2020/ Instituto Nacional de Estadística y Geografía (INEGI). (22 de marzo de 2021b). Estudio sobre la Demografía de los Negocios EDN 2020. Segundo Conjunto de Resultados. Obtenido de Instituto Nacional de Estadística y Geografía: https://www.inegi.org.mx/contenidos/programas/edn/2020/doc/EDN2020Pres2.pdf Instituto Nacional de Estadística y Geografía (INEGI). (31 de mayo de 2021c). ECOVID-IE. Encuesta sobre el impacto económico generado por COVID-19 en las empresas. Resultados tercer evento. Obtenido de Instituto Nacional de Estadística y Geografía: https://www.inegi.org.mx/contenidos/programas/ecovidie/doc/PRECOVIDIER3.pdf

Kahveci, E. (mayo de 2021). Surviving COVID-19 and beyond: a conceptual framework for SMEs in crisis. Obtenido de Verslas: Teorija ir Praktika. Vilnius Gediminas Technical University: https://journals.vgtu.It/index.php/BTP/article/view/13020

Lombardi, C. V., Chidiac, N. T., \& Record, B. C. (25 de junio de 2021). Starbucks coffee corporation's marketing response to the COVID-19 pandemic. Obtenido de Innovative Marketing , 17(2), 177-188:

https://www.businessperspectives.org/images/pdf/applications/publishing/templates/article/as sets/15206/IM_2021_02_Lombardi.pdf

Loxton, M., Truskett, R., Scarf, B., Sindone, L., Baldry, G., \& Zaho, Y. (30 de julio de 2020). Consumer Behaviour during Crises: Preliminary Research on How Coronavirus Has Manifested Consumer Panic Buying, Herd Mentality, Changing Discretionary Spending and the Role of the Media in Influencing Behaviour. Obtenido de Risk Financial Managament. 13(8), 166: https://doi.org/10.3390/jrfm13080166

Meghisan-Toma, G.-M., \& Cosmin, N. V. (26 de julio de 2020). ICT Security Measures for the Companies within European Union Member States - Perspectives in COVID-19 Context. Obtenido de Proceedings of the International Conference on Business Excellence, vol.14, no.1, 2020, pp.362-370.: https://doi.org/10.2478/picbe-2020-0035

Nilasari, B. M., Nisfiannoor, M., \& Devinta, F. R. (Junio de 2021). Changes in Motivation That Affect Employee Performance during the Covid 19 Pandemic. Obtenido de Jurnal Aplikasi Manajemen, Volume 19, Number 2, Pages 435-447: http://dx.doi.org/10.21776/ub.jam.2021.019.02.19

Onour, I. A. (junio de 2021). The impact of COVID-19 pandemic shock on major Asian stock markets: evidence of decoupling effects. Obtenido de Monetary Economics 34 (2), 21-32.: https://statecounsellor.files.wordpress.com/2021/06/210203.pdf

Stoica, O. C. (diciembre de 2020). AN EARLY EVIDENCE ON CSR REPORTING IN THE CONTEXT OF COVID-19. Obtenido de Journal of Public Administration, Finance and Law: 
https://www.jopafl.com/uploads/issue18/AN_EARLY_EVIDENCE_ON_CSR_REPORTING_IN _THE_CONTEXT_OF_COVID19.pdf

Secretaría del Trabajo y Previsión Social (STPS). (15 de julio de 2021). Puestos de Trabajo registrados en el IMSS. Obtenido de Secretaría del Trabajo y Previsión Social: http://siel.stps.gob.mx:303/ibmcognos/cgibin/cognos.cgi?b_action=cognosViewer\&ui.action=run\&ui.object=XSSSTART ${ }^{\star} 2$ fcontent ${ }^{\star} 2$ ffol

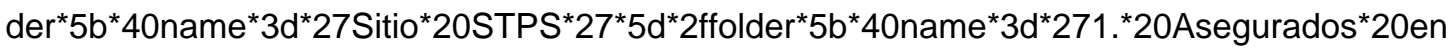
*20el*20IMSS*27*5d*2freport*5b*40name*3d*27Tot

Suswati, E. (junio de 2021). Work Placement Affects Employee Performance through Work Motivation. Obtenido de Jurnal Aplikasi Manajemen, Volume 19, Number 2, Pages 394-403. Malang: Universitas Brawijaya: http://dx.doi.org/10.21776/ub.jam.2021.019.02.15.

Verastegui, T. F., \& Vargas, M. J. (2021). Estrategias de Merchandising:un análisis de su efectividad para la atracción de nuevos clientes. Obtenido de Revista Academia \& Negocios Vol.7 (1) pp. 41 -54: https://revistas.udec.cl/index.php/ran/article/view/3067/3180

Wardhani, R. S., Elvany, R. E., \& Awaluddin, M. (mayo de 2021). Banking financial performance during Covid-19. Obtenido de Technium Social Sciences Journal, vol 19(1), 302-310. : https://techniumscience.com/index.php/socialsciences/article/view/3194/1233 